\title{
Internet Geographic Information Security Supervision Platform Architecture Research
}

\author{
http://dx.doi.org/10.3991/ijoe.v9iS2.2562 \\ CHEN Wanzhi ${ }^{1,2}$, LIU Jiping ${ }^{2}$ and WANG Yong ${ }^{2}$ \\ ${ }^{1}$ Liaoning Technical University, FuXin, Liaoning Province, P.R.China \\ ${ }^{2}$ Chinese Academy of Surveying and Mapping, BeiJing, P.R.China
}

\begin{abstract}
Nowadays every link of geographical information from products manufacture, management, and distribution to application would be faced with tremendous security risks, which urgent need to establish a geography information security management and monitoring system with unified and multilevel action manner by the new technology and information measures. This article presents the overall architecture of the platform and typical services node, based on analysis of construction requirements for the Internet geographic information security supervision platform, laid the theory ground work for further research of the final geographic information security management measures and technique adapt to the development of science technology level and the requirements of social widely, and realized the overall aim of geography information security supervision in the end.
\end{abstract}

Index Terms - Internet, Geographical Information, Security Supervision, Platform Architecture.

\section{INTRODUCTION}

With the rapid development of our surveying and mapping cause, the geographical information industry developed rapidly and geographic information resources are continuously enriched, which has been playing an increasingly important role in the national economy, social development and national defense construction. The rapid popularization of Internet and cloud computing, Internet of things, etc. which new information and modern surveying and mapping technology has been developed rapidly, those making people could be efficiently to acquire and use a variety of the geographical information, at the same time, following a serious challenge of the geographical information safe at digital environment from production, management and approval to release and application. Users can easily obtained geographical information of high precision image and many sensitive areas through online service of the geographical information, also tagging on the map however their interest in the information by tag services features. The security problems for geographic information services have become increasingly prominent. Relevant departments of the country has always attached great importance to the geographical information safe of production, management and supervision of Internet web map, geographic information system services site. However, compare with higher maturity of Internet information security technologies and products, we still lack geographic information security management measures and technique, which adapt to the development of science technology level and the requirements of social widely. And foreign developed countries had set up special organizations to strengthen the geographical information resources collected and protected, such as "National Geospatial-Intelligence Agency" (NGA) for the US government, the same level with FBI, CIA and NSA, specialized responsible for the important geographical information collecting and supervision.

Geographical information is state infrastructure and strategic information resource, involved in national economic, political and military secrets and sensitive information. Thus aim to every link of geographical information products manufacture, management, distribution and application would be faced with tremendous security risks, which urgent need to establish a geography information security management and monitoring system with unified and multilevel action manner by the new technology and information measures, realized the overall process of supervision informationize for geographical information products manufacture, management, distribution and application, improving comprehensive ability of the map and geographic information credible, controlled and security in the course of management, distribution and services, and then the rapid respond ability of Internet (the Internet of things) geographic information security supervision come into being, set up more authorities cooperative mechanism of the geographical information security supervision, provide administrative law enforcement support for Internet maps and geographical information lawbreaking behavior, ensure the national geographic information security. The geographical information security management project mainly including two kinds of development task, the secret-related geographical information security and the Internet (the Internet of things) geographical information security supervision. This article expatiates the research basis of the second field.

\section{THE CONSTRUCTION REQUIREMENTS AND OVERALL ARCHITECTURE}

The Internet geographical information security supervision is a collaboration work that need more administrative authorities and department in different regions to completed commonly, mainly responsibility for the administrative departments of surveying and mapping, the division of labour for relevant departments according to law, cooperate perfectly and set up a shortcut cooperative mechanism. In the end, supervision of Internet map and network geographical information service standardized and institutionalized, information shared and supervision profession streamlined, consist of to draw up 


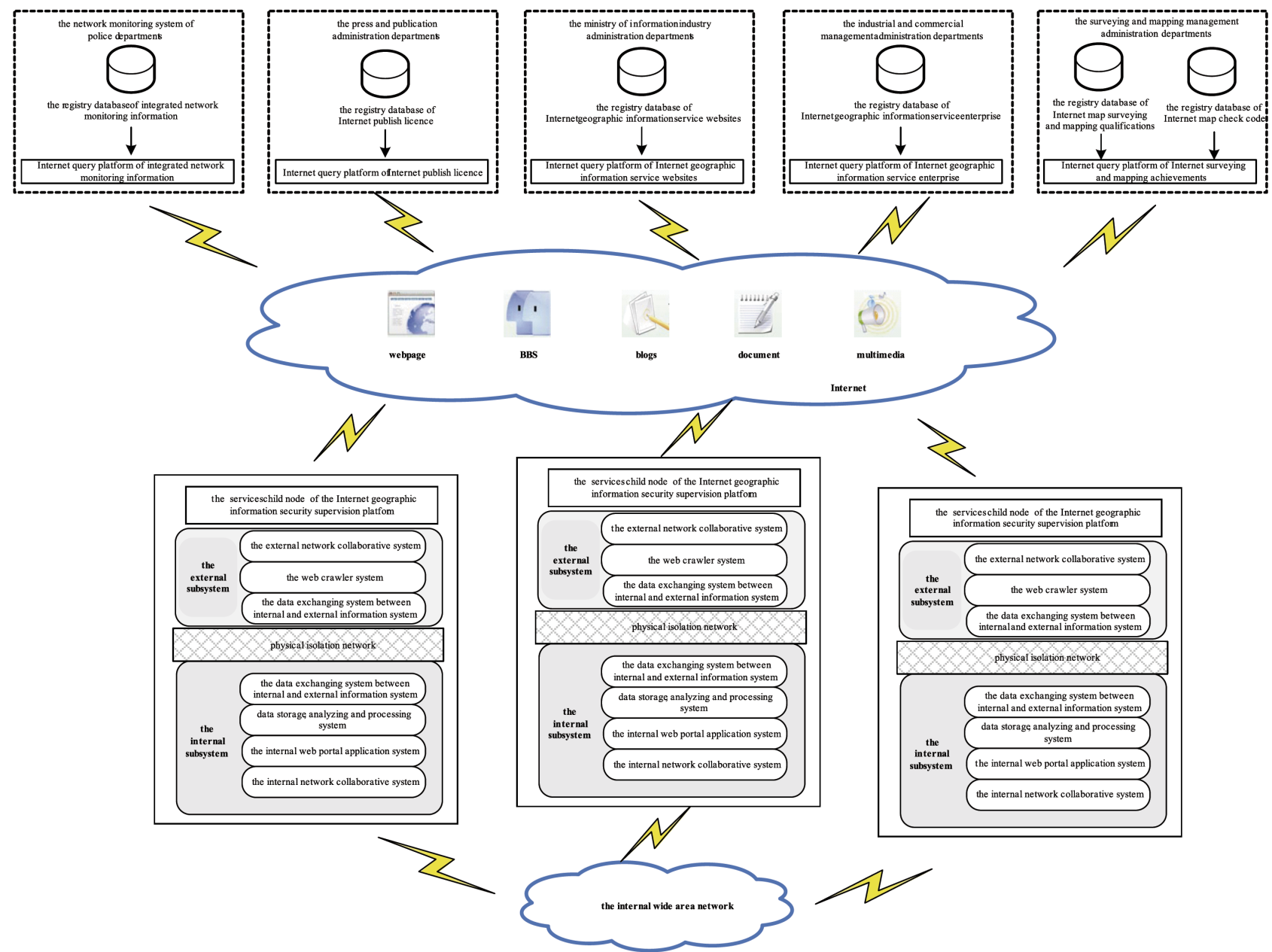

Figure 1. The overall architecture of the geography information security supervision platform.

the directory system of secret-related geographical information security, the security level of Internet geographical information security, the approved standard of networked map and geographical information; to establish the standard system of Internet geographical information security supervision perfectly; to give great impetus to construction of the map and geographical information approved network system; then to realized the approved procedure management networked for achievements in surveying and mapping, efficient identification and semi-automatization checked for important geographical element and secret-related geographical information by cooperative approved for map and intellectual processing technology, such as semantic analysis and pattern recognition; and in another hand, to accelerate the construction of Internet geographical information security supervision; to strengthen monitoring routinely of key point site for map and geographical information service, handle basic tasks: automatic discovery for Internet secret-related geographical information, intelligence identification, constantly tracked, investigate and collect evidence, analysis and evaluation, etc. So next to introduce primary service for the Internet geographic information security supervision platform in details.

\section{A. The Capability of Discovery and Identification for Internet Geographical Information Service Site}

The system through the Internet search engine scanning continuous, found them who provided geographical information service site, then remarked and archiving into database. Checking the civil sites' ICP/IP with the Internet recorded system of Ministry of Industry and Information Technology, at same time, making approval examine and verify of geographical information system more strictly, and recording all related items.

\section{B. The Capability of Automated Acquisition and Analysis for Internet Geographical Information}

The system analysis the content of the geographical information services site based on the acquired site records database, collect the effective information, and then place on formatted file.

\section{The Capability of Intelligence Identification, \\ Constantly Tracked and Take the Evidence, Signature \\ Analysis for Secret-related Geographical Information}

The system analysis the content of related to national security for politics, military, major objective, energy sources, etc., from acquired information by relevant 


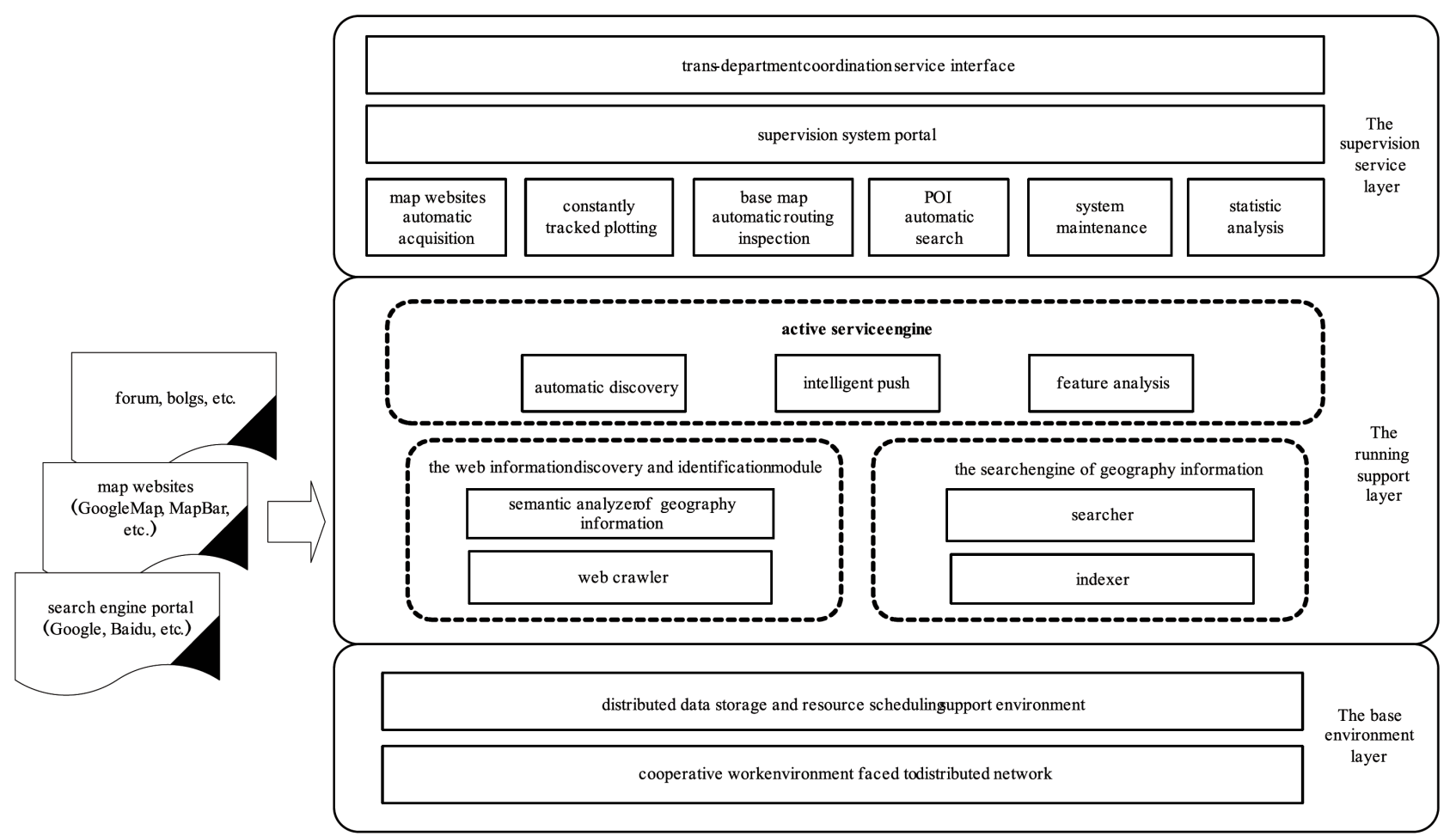

Figure 2. The typical services node architecture of the Internet geographic information security supervision platform.

analysis methods. Then relevance content would be recorded and marked, for subsequent information retrieval.

\section{The Capability of Efficient Management, Sustainable Service and Quick Reaction}

The system could acquire effective information effectively; the system maintainer could manage the whole system maintenance and setting easily, and control system application through short-term training. And the system could respond to operations of administrator rapidly, master the system current running condition in time. The system's redundant configuration could recovery from accidental interrupted situation.

\section{E. The Capability of Linked Up and Down, Cooperate with Other Departments, Support Administrative Law Enforcement}

The system record database could distribute shared for multi-level interactions, different levels and different departments by networked and operational collaboration. Legitimate users can easily log in platform system to extract relevant information, to provide evidence of law enforcement.

As suggested above, according to the national geographic information public services platform three levels construction requirement - shared, distributed and online; the basic principles - "makes overall plans, cent pace is carried out; overall planning and guidance, decentralized management”; the construction requirements for the Internet geographic information security supervision platform. The system platform's general architecture support by the newest computer network integration technology, which face to each department supervision on web maps and geographic information service websites for Internet with distributed B/S architecture, as shown in Fig.1. The information resources of platform derived from the each department network retrieval service, which use local distributed database, shared but isolated model between intranet and extranet. In fact, the local distributed database independent maintenance by each department except provided the information interface classes to the platform, not other sensitive data, each department could achieve the websites supervise object relevant details through retrieve function for the platform fast. This model more conducive to cooperation and distribution of responsibilities, sharing information between the multiindustry departments.

\section{The TypicAl SERVICES Node ARChITECtURE}

The centre of Internet geographic information security supervision in the overall architecture is the services node with distributed, redundant and shared function for platform; consist of three-layers construction - base environment, running support and supervision service, as shown in Fig.2. The function of the typical services node as follows.

\section{A. The Web Crawler System}

To multiple sources and heterogeneous information for geographic information service from webpage, forum, blogs, etc. of Internet, the system collect and store to related information with distributed multi-agent crawler network software, centralized logic and distributed physics search strategy. Which support to collect the spatial information automatically, classify and file them, including maps, vector data, POI(Point Of Interest) layer, geographical name and address, tag of user, map service, text, photograph, etc. 


\section{B. The Data Exchanging System between Internal and External Information System}

The system realized data safety transmission with "ferry" operating mechanism, which must be internal and external network data security, dynamic and sustained in physical isolation network environment.

\section{The Collaborative System between Internal and External Network}

The system support the geographical information security exchange function between all WANs levels of nodes, dynamic and linkage; support the online or off-line mode data security exchange function between all supervision service node; support data exchange and collaborative function between all service node. At the same time, real-time monitoring all node system's services, security, running, etc.; recording all node system's running key information and log; alarm processing in the emergency; collecting popular feelings.

\section{The Data Storage, Analyzing and Processing System}

The system support efficient storage and fast scheduling for vast quantities of the Internet heterogeneous information by distributed storage mechanism, and data backup and storage management. Then the system analyzing and processing the Internet heterogeneous information on the basis of the structure characteristic for all classes of geographic information, support splitting and polymerizing plotting function for KML format file, and for point, line, plane object fast, rapid assessment function for secret-related content, support to identification of secret-related object from geographic information, collect the space-locating information, so that the geographical positions, objects, spatial attributes could be lot size automatic identification, collection and achievement management. In the end, the system could automatic discovery, automatic mark, and automatic alarm for secret-related content on the basis of semantic feature of secret-related geographic information.

\section{E. The Internal Web Portal Application System}

The system aim to routine geographic information security supervision, support to core application service function by web portal, mainly including: constantly tracked for Internet information; automatic alarm and collect evidence for secret-related geographic information; administrative supervision, and SMS presentation, mail alarm, Word/Excel import or export, automatic generation for official document or report forms, etc.; integrated with OA system; content statistic analysis by time, space, subject, etc. and user behavioral analysis, and forecast.

For the core function of supervision, the main parts are the web crawler system, data storage, analyzing and processing system, and internal web portal application system. The technicality key and difficult point are the automated acquisition and analysis for Internet geographical information, distributed storage and scheduling. Refer to the distributed cluster acquisition, multithreading concurrency scanning, real-time monitoring, metadata extraction, automatic classification, repetitive computation, automatic index, automatic keywords extraction, intelligent search and retrieval system in database, veridical search application, network popular feelings monitoring, special topic database, etc.

\section{CONCLUSIONS}

This article presents the overall architecture of the platform and typical services node, based on analysis of construction requirements for the Internet geographic information security supervision platform, laid the theory ground work for further research of the final geographic information security management measures and technique adapt to the development of science technology level and the requirements of social widely, and realized the overall aim of geography information security supervision in the end.

\section{ACKNOWLEDGMENT}

This work was supported in part by Hi-Tech Research and Development Program of China (863) under Grant Nos. SS2012AA120702.

\section{REFERENCES}

[1] Surveying and Mapping Development Strategy Research Group for State Bureau of Surveying and Mapping, "Research report of Surveying and Mapping Development Strategy", unpublished.

[2] LIU Jiping, et al. "Research report of the geography information security supervision platform construction", unpublished.

[3] HAN Quanwei, "Thinking on Establishing National Internet and Web Geographic Information Services Supervision Platform", Geomatics \& Spatial Information Technology, vol. 32(6), pp. 7073, Dec 2009.

[4] Jia Lin, Alexander Halavais, Bin Zhang, "The Blog Network in America: Blogs as Indicators of Relationships among US Cities", CONNECTIONS, vol. 27(2), pp. 15-23, 2007

\section{AUTHORS}

CHEN Wanzhi received his B.Sc. and M.Sc. degree in electrical engineering from Liaoning Technical University, FuXin, Liaoning Province, P.R.China. Now he is a $\mathrm{PhD}$ candidate of Chinese Academy of Surveying and Mapping.

His main research interests include GIS and information security. He have participated in several projects include two National Natural Science Foundations of China and one National Grand Fundamental Research 863 Program of China and published more than 10 research papers from 2007(e-mail: chenwanzhi@lntu.edu.cn).

LIU Jiping received an M.S. degree from WuHan Technical University of Surveying and Mapping, WuHan, China, in 1992 and a Ph.D. degree from Information Engineering University of the People's Liberation Army, ZhengZhou, China, in 2004. Now he is a Research Prof and $\mathrm{PhD}$ supervisor at Chinese Academy of Surveying and Mapping. His research interests cover GIS of Egovernment and Spatial Data Mining and information security.

WANG Yong received an M.S. degree from Chinese Academy of Surveying and Mapping. Now he is a Assistant Prof at Chinese Academy of Surveying and Mapping. His research interests cover GIS of Egovernment and Spatial Data Mining and information security.

This article is an extended and modified version of a paper presented at the International Conference on Mechanical Engineering, Automation and Material Science (MEAMS2012), held 22-23 December 2012, Wuhan, China. Received 14 February 2013. Published as resubmitted by the authors 25 March 2013. 